\title{
Uma Revisão do Uso de Processos Oxidativos Avançados para Descoloração de Águas Residuais de Efluentes
}

\author{
Letícia G. Oliveira, Fabrício H. Fernandes, Weber D. Mesquita, Mario \\ G. Junior, Maria R. de C. Santos \& Maria F. do C. Gurgel
}

A preocupação com o meio ambiente e o modo correto de descarte de corantes têxteis em efluente têm sido tema de vários estudos. A contaminação de águas por corantes industriais é, sem dúvida, um dos problemas atuais não só no Brasil, mas em todo mundo. O objetivo dos pesquisadores acerca deste tema envolve o estudo de Processos Oxidativos Avançados (POAs) que se apresentam como uma potente alternativa de método rápido e eficiente para a completa descoloração de corantes em águas. Neste estudo, realizou-se uma revisão da literatura em torno dos mais novos métodos de descoloração de corantes têxteis.

Palavras-chave: descoloração; POAs; corantes.

Concern about the environment and the correct way of disposal of textile dyes in effluents, has been the subject of several studies. Water contamination by industrial dyes is undoubtedly one of the current problems, not only in Brazil, but worldwide. The aim of the researchers on this subject involves the study of Advanced Oxidation Process (AOPs) that present as a quick and efficient alternative to the complete discoloration of dyes in water. In this study, a review of the literature on the newest methods of discoloration of textile fabrics is performed.

Keywords: discoloration; AOPs; dyes. 


\section{Introdução}

$\mathrm{O}$ aumento da população mundial a cada ano que passa, como consequência, traz aumento excessivo de descarte de resíduos industriais. Assim, impõe a necessidade de novos métodos para a despoluição de rios, lagos e as águas residuais industriais. A utilização de corantes pelas indústrias têxteis e de alimentos tem aumentado gradativamente com o passar dos anos. Em indústrias têxteis, o processo de tingimento, utiliza grande quantidade de corantes e muita água limpa. Após este processo, uma grande quantidade dessa solução ainda concentrada é descartada de forma inadequada ${ }^{1}$.

Vários processos têm sido utilizados para a remoção de corantes de águas, dentre eles podem-se citar: adsorção com carvão ativado; decomposição enzimática e processos microbiológicos. Uma grande desvantagem destes processos é o alto custo operacional e em alguns casos a baixa eficiência com longos tempos de tratamento. Neste sentido, vem sendo crescente, nos últimos anos, a busca de materiais, como catalisadores, que permitam a fotodegradação destes no meio ambiente ${ }^{2}$.

As águas residuais contêm até $50 \%$ dos corantes adicionados inicialmente e $100 \mathrm{~g} / \mathrm{L}$ de sais ${ }^{3,4}$, resultando em efeitos cancerígenos graves na biota aquática e nos seres humanos ${ }^{5,6}$. Além da cor, ele apresenta também alteração no $\mathrm{pH}$ final, características de biodegradabilidade e variação de vazão. Desta forma, o tratamento adequado das águas residuais geradas pelas indústrias têxteis tornase imprescindível para a sustentabilidade dos ecossistemas.

Vários estudos mostram que o consumo médio de água no processo produtivo na indústria têxtil varia entre 50 a $100 \mathrm{~L} / \mathrm{kg}$. Esse alto consumo advém, principalmente, das operações de lavagem e beneficiamento de fios e tecidos, além da lavagem das plantas industriais com os equipamentos?. Alguns corantes são resistentes |à degradação com algumas técnicas. Algumas técnicas convencionais como os processos biológicos, a filtração por membranas, a coagulação, a adsorção e a troca iônica, são bastante utilizadas, porém novos métodos alternativos estão sendo investigados ${ }^{8}$. Dentre os processos alternativos, os chamados Processos Oxidativos Avançados (POAs) têm se mostrado uma alternativa interessante para o tratamento desses efluentes. Os POAs são baseados na produção in situ do radical hidroxila, que pelo fato de apresentar um alto potencial padrão de redução $(2,73 \mathrm{~V}$ vs EPH), ou seja, alta capacidade oxidativa, é utilizado para promover a degradação de diversas moléculas orgânicas ${ }^{9}$.

Dentre os POAs, existem vários métodos de geração de radical hidroxila. Na Tabela 1, estão representados os principais tipos de POAs encontrados comumente.

Tabela 1: Sistemas típicos de Processos Oxidativos Avançados.

\begin{tabular}{|c|c|c|}
\hline Processo & Homogêneo & Heterogêneo \\
\hline \multirow{4}{*}{ Com irradiação } & $\mathrm{O}_{3} / \mathrm{UV}$ & \\
& $\mathrm{H}_{2} \mathrm{O}_{2} / \mathrm{UV}$ & \multirow{2}{*}{$\mathrm{O}_{3} / \mathrm{H}_{2} \mathrm{O}_{2} / \mathrm{UV}$} \\
& Foto-Fenton & \\
\hline \multirow{3}{*}{ Sem irradiação } & & \\
& $\mathrm{O}_{3} / \mathrm{HO}-$ & \\
& $\mathrm{O}_{3} / \mathrm{H}_{2} \mathrm{O}_{2}$ & $\mathrm{O}_{3} /$ catalisador \\
& Reativo de Fenton & \\
\hline
\end{tabular}

Podem-se mencionar algumas implicações dos Processos Oxidativos Avançados, tais como ${ }^{10-13}$ :

- Os POAs produzem alterações químicas na estrutura dos corantes, podendo inclusive levar à sua completa mineralização.

- Os POAs podem ser utilizados no tratamento de contaminantes cuja concentração seja muito baixa, até nas concentrações de $\mathrm{ppb}$.

- Na maioria dos casos, os POAs não geram resíduos, com exceção de alguns processos que podem envolver precipitação (por exemplo, óxidos férricos).

- Assim como qualquer outro tratamento, a Oxidação Avançada não pode ser utilizada a qualquer tipo de resíduo.

- Os gastos para a mineralização podem ser altos, principalmente, devido ao custo de energia;

- Pode haver produção de subprodutos de reação indesejados, alguns podem ser até mais tóxicos que o resíduo;

- Apresentam restrições de aplicação em condições de elevada concentração dos poluentes; 
- Dentre os processos de geração do radical hidroxil, destacam-se os sistemas que utilizam ozônio, peróxido de hidrogênio, a Fotocatálise Heterogênea e Via Reação de Fenton e Foto-Fenton.

\section{OZÔNIO}

Uma alternativa que vem sendo estudada mais a fundo para o tratamento de efluentes residuais no POA é o ozônio, principalmente, técnicas acopladas como $\mathrm{O}_{3} / \mathrm{UV}$, $\mathrm{O}_{3} / \mathrm{H}_{2} \mathrm{O}_{2}, \mathrm{O}_{3} / \mathrm{TiO}_{2}$, e vem revelando ótimos resultados na descontaminação destes efluentes ${ }^{14}$.

Frente a isso, existem 3 diferentes técnicas para produzir o ozônio:

a. Exposição do $\mathrm{O}_{2}$ à luz ultravioleta,

b. Eletrólise do ácido perclórico e

c. Descarga eletroquímica.

Todos métodos vêm sendo pesquisados nos últimos anos, porém o mais utilizado pelos ozonizadores é o que emprega-se a descarga elétrica, pois gera maior quantidade de ozônio com menor custo, que também é conhecido como efeito corona (efeito provocado por uma carga elétrica produzida pela ionização nas redondezas de um condutor quando o gradiente elétrico excede um valor não suficiente para causar um arco elétrico). Esse método consiste em obter o ozônio pela passagem de ar ou oxigênio puro entre dois eletrodos submetidos a uma alta diferença de potencial ${ }^{15}$.

O ozônio é instável em água. A decomposição do ozônio em águas naturais é caracterizada por uma rápida diminuição da concentração inicial do ozônio, seguida de uma segunda fase na qual a concentração de ozônio diminui, seguindo uma cinética de primeira ordem, sendo que o principal produto de decomposição do ozônio é o radical hidroxila $\mathrm{OH} \bullet$. Dependendo da qualidade do meio em que se encontra, o tempo de meia vida do ozônio varia de alguns segundos até horas. Existem inúmeras condições para a estabilidade do ozônio, dentre eles, o pH merece especial atenção, uma vez que os íons hidroxila iniciam o processo de decomposição do ozônio ${ }^{16}$, como mostrado nas equações de 1-2:

$$
\begin{gathered}
\mathrm{O}_{3}+\mathrm{OH}^{-} \rightarrow \mathrm{HO}_{2}^{-}+\mathrm{O}_{2} \\
\mathrm{O}_{3}+\mathrm{HO}_{2}^{-} \rightarrow \mathrm{OH} \bullet+\mathrm{O}_{2}^{-} \cdot+\mathrm{O}_{2}
\end{gathered}
$$

Galvão e colaboradores ${ }^{17}$ aplicaram a técnica combinada ozônio e processo oxidativo avançado (POAs) no tratamento de remoção de cor em águas residuárias têxtil e avaliou sua eficiência, realizando ensaios e o processo piloto simulando o tratamento do efluente têxtil em laboratório. Após o tratamento os resultados possibilitaram concluir que houve uma remoção significante de cor em 58,24\%, DQO de $44,75 \%$ e turbidez em $65,43 \%$ no tempo de 90 min, comprovando que o método do ozônio é realmente satisfatório.

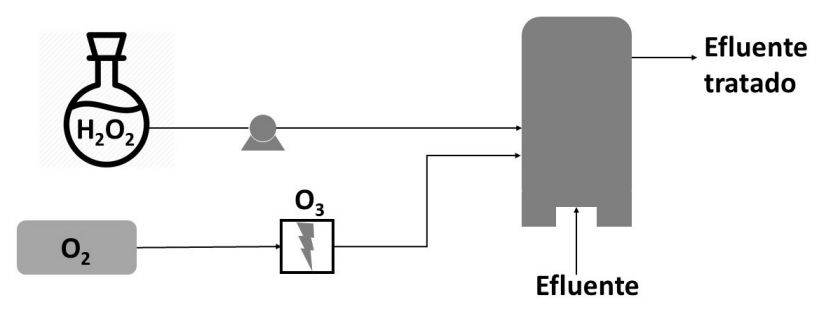

Figura 1: POAs combinado de ozônio e peróxido de hidrogênio

\section{PERÓXIDO DE HIDROGÊNIO}

Outro processo utilizado nos estudos é a utilização de peróxido de hidrogênio para a descoloração de águas naturais. Este processo que utiliza $\mathrm{H}_{2} \mathrm{O}_{2} / \mathrm{UV}$ pode levar à degradação completa e à conversão para $\mathrm{CO}_{2}$ e $\mathrm{H}_{2} \mathrm{O}$ e sais inorgânicos da maioria dos contaminantes orgânicos. A fotólise do $\mathrm{H}_{2} \mathrm{O}_{2}$ pela radiação $\mathrm{UV}$ gera dois radicais hidroxila, que agem degradando a matéria orgânica e formando compostos mais simples. Quando o $\mathrm{H}_{2} \mathrm{O}_{2}$ está em excesso pode ocorrer uma reação paralela, que diminui a razão de degradação da matéria orgânica, sendo assim, é de suma importância analisar a quantidade do reagente ${ }^{18}$. Isso ocorre porque $\mathrm{o}_{2} \mathrm{O}_{2}$ em excesso age capturando radicais hidroxilas, como observa-se nas equações de 3-5:

$$
\mathrm{H}_{2} \mathrm{O}_{2}+\mathrm{h} v \rightarrow 2 \mathrm{OH} \bullet
$$


$\mathrm{R}-\mathrm{H}+\mathrm{OH} \bullet \rightarrow$ Produtos finais $\left(\mathrm{CO}_{2}, \mathrm{H}_{2} \mathrm{O}, \mathrm{NO}_{3}{ }^{-}, \mathrm{Cl}^{-}\right)$

$\mathrm{OH} \bullet+\mathrm{H}_{2} \mathrm{O}_{2} \rightarrow \mathrm{HO}_{2} \bullet+\mathrm{H}_{2} \mathrm{O}$

A combinação de reagente peróxido de hidrogênio com a radiação ultravioleta é muito mais poderosa, pois resulta em uma reação indireta. Isso se dá devido ao fato do potencial de oxidação do radical hidroxil $\left(\mathrm{E}_{0}=+2,80 \mathrm{~V}\right)$ ser mais alto que o do peróxido de hidrogênio molecular $\left(\mathrm{E}_{0}=+1,78 \mathrm{~V}\right)$, podendo, assim, promover oxidação mais enérgica e mais efetiva ${ }^{11}$.

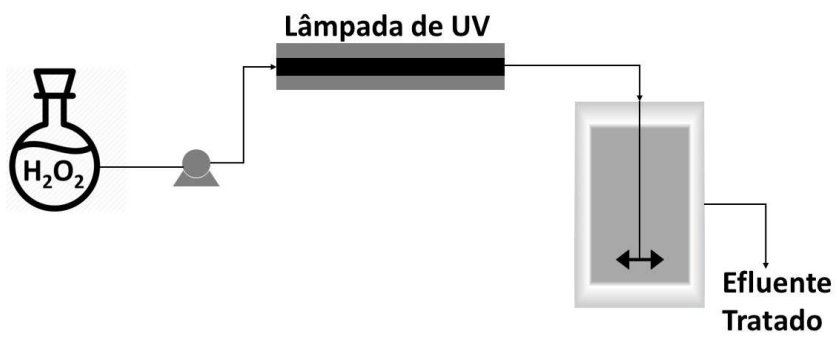

Figura 2: POA do peróxido de hidrogênio

Marmitt e colaboradores fizeram a avaliação da aplicação de processo de fotólise direta aplicando H2O2/ UV, no tratamento de um efluente sintético constituído de corantes alimentícios, sendo que este chegou a reduzir a coloração do efluente em $96,4 \%$ e a DQO em $38,56 \%$ na presença de oxidante $\mathrm{H} 2 \mathrm{O} 2$, ou seja, mostrando uma ótima descoloração do efluente ${ }^{19}$.

\section{FOTOCATÁLISE HETEROGÊNEA}

Com a finalidade de desenvolver células fotoeletroquímicas, começaram a produzir novos combustíveis a partir de materiais mais baratos, visando a transformação de energia solar em química. Assim foi que começaram a origem dos processos de Fotocatálise Heterogênea por volta dos anos $70^{11}$.

No processo heterogêneo, têm-se semicondutores como catalisadores na fase sólida e a reação química ficam ocorrendo na área de interação entre as duas fases e a velocidade depende de fatores tais como: área superficial de contato, da concentração do material, temperatura, pressão, etc $^{20}$. Esses semicondutores são ativados através da luz solar ou de uma luz artificial, provocando uma transição eletrônica.

A Fotocatálise Heterogênea consiste num processo onde os elétrons, após a excitação pela irradiação, passam da banda de valência para a banda de condução e ocorre a geração de sítios oxidantes e redutores capazes de catalisar as reações químicas, oxidando os compostos orgânicos e produzindo $\mathrm{CO}_{2}$ e $\mathrm{H}_{2} \mathrm{O}$. A região entre as duas bandas é chamada de "bandgap". Na degradação dos compostos orgânicos via fotocatálise heterogênea pode ocorrer por várias maneiras de serem oxidadas. As moléculas de água adsorvida na interface do material com a atividade fotocatalítica, reagem com as vacâncias formadas na banda de valência, gerando os radicais hidroxilas fortemente reativos que podem reagir com as moléculas orgânicas no meio. Os oxigênios presentes no meio podem reagir com os elétrons na banda de condução produzindo $\mathrm{O}^{2-}$, que é uma espécie reativa, podendo assim oxidar mais compostos orgânicos. Assim, o bom fotocatalisador será aquele que viabilizará esses dois processos ocorrendo simultaneamente ${ }^{21}$. O esquema abaixo mostra essa fotoativação:

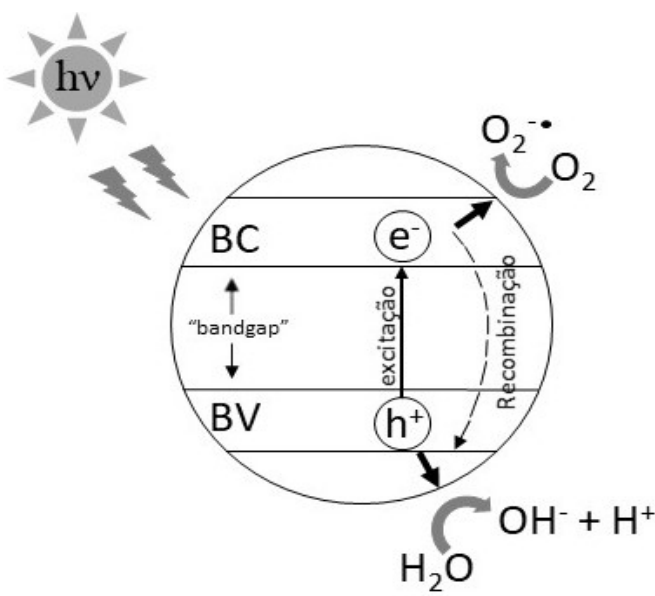

Figura 3: Sistema representativo da foto-ativação de semicondutor

Rodrigues e colaboradores ${ }^{22}$ sintetizaram os compostos de Ce1-xInxO2 e estudaram suas propriedades fotocatalíticas heterogêneas frente a degradação do corante 
de azul de metileno e concluiu que cerca de $52 \%$ de corante foi fotodegradado, com a utilização do catalisador, após 120 minutos sob iluminação UV-Vis.

Giraldi e colaboradores ${ }^{13}$ obtiveram partículas de $\mathrm{ZnO}$ dopado com manganês e avaliaram o potencial fotocatalítico frente à fotodegradação do corante azul de metileno. Obtiveram como resultado na amostra $\mathrm{ZnO}: 0,25 \mathrm{Mn}$, uma decoloração de $78 \%$ em $150 \mathrm{~min}$, e concluíram que a atividade fotocatalítica foi satisfatória.

Pereira e colaboradores ${ }^{23}$ utilizaram o composto HY340R (ácido de nióbio) para avaliar a atividade fotocatalítica do material com e sem tratamento hidrotérmico, e verificouse que as partículas submetidas a tratamento térmico permitiu a degradação de praticamente $100 \%$ do corante de azul de metileno em $150 \mathrm{~min}$, enquanto no experimento realizado com as partículas sem tratamento térmico a degradação foi de $87 \%$.

$\mathrm{Na}$ literatura, existem vários trabalhos que utilizam o processo de fotocatálise heterogênea, e cada vez mais, buscam-se novos materiais alternativos para aumentar a atividade de descoloração frente aos corantes em geral ${ }^{24-28}$

\section{VIA REAÇÃO DE FENTON E FOTO-FENTON}

Na Reação de Fenton utiliza-se um catalisador a base de ferro e emprega o peróxido de hidrogênio para a geração de radicais hidroxilas, espécie essencial para a oxidação de compostos orgânicos, que é catalisada por íons ferro ou íons férrico, em meio ácido (equação 6) 2,29.

$$
\mathrm{H}_{2} \mathrm{O}_{2}+\mathrm{Fe}^{2+}+\mathrm{H}^{+} \rightarrow \mathrm{OH}^{-}+\mathrm{OH} \bullet+\mathrm{Fe}^{3+}+\mathrm{H}^{-}
$$

Os íons ferrosos $\left(\mathrm{Fe}^{2+}\right)$ agem como catalisadores para a decomposição do peróxido de hidrogênio em meio ácido, gerando o radical hidroxila. A degradação ocorre de maneira direta, em que íons $\mathrm{Fe}^{2+}$ e $\mathrm{Fe}^{3+}$ podem reduzir ou oxidar diretamente a matéria orgânica, e indireta, onde há a formação do radical hidroxila ${ }^{12}$.

$$
\begin{aligned}
& \mathrm{Fe}^{2+}+\mathrm{Cl}-\mathrm{RH} \rightarrow \mathrm{Fe}^{3+}+\mathrm{Cl}^{-}+\cdot \mathrm{RH} \\
& \mathrm{Fe}^{3+}+\mathrm{R} \rightarrow \mathrm{Fe}^{2+}+\mathrm{R}^{+}
\end{aligned}
$$

$$
\mathrm{OH} \bullet+\mathrm{R} \rightarrow \mathrm{Fe}^{2+}+\mathrm{R}^{+}
$$

$\mathrm{O}_{2} \mathrm{O}_{2}$ pode assegurar o radical hidroxila, formando o radical hidroperoxil $\left(\mathrm{HO}_{2}{ }^{-}\right)$, que possui menor potencial de redução $\left(\mathrm{E}_{0}=1,42 \mathrm{eV}\right)$ que o radical hidroxila, prejudicando assim o processo de degradação.

$$
\mathrm{H}_{2} \mathrm{O}_{2}+\mathrm{OH} \bullet \rightarrow \mathrm{HO}_{2} \cdot+\mathrm{H}_{2} \mathrm{O}
$$

A potencialidade do uso desse processo para o tratamento de efluentes se deve principalmente à sua simplicidade, uma vez que a reação ocorre à temperatura e à pressão ambiente e se aplica a uma grande variabilidade de compostos, além de o ferro ser o quarto elemento mais abundante da crosta terrestre ${ }^{11}$.

Nos anos 80, foi descoberto o processo Foto-Fenton, diferentemente do Fenton, em que o Foto-Fenton ocorre pela absorção da radiação ultravioleta (UV). Ele provoca a fotorredução dos íons $\mathrm{Fe} 3+$ previamente formados, com geração de mais de um mol do radical hidroxila ${ }^{30,31}$. Assim como no processo Fenton, os radicais hidroxila formados no Foto-Fenton reagem destruindo os poluentes.

$$
\begin{aligned}
& \mathrm{Fe}^{3+}+\mathrm{H}_{2} \mathrm{O}_{2}+\mathrm{hv}(\mathrm{UV} \text { ou Vis }) \rightarrow \mathrm{Fe}^{2+}+\mathrm{H}^{+}+\mathrm{OH} \bullet \\
& \mathrm{Fe}^{3+}+\mathrm{H}_{2} \mathrm{O} \rightarrow \mathrm{Fe}(\mathrm{OH})^{2+}+\mathrm{H}^{+} \\
& \mathrm{Fe}(\mathrm{OH})^{2+}+h v \rightarrow \mathrm{Fe}^{2+}+\mathrm{OH} \bullet \\
& \mathrm{OH} \bullet+\mathrm{H}^{+}+\mathrm{e}-\rightarrow \mathrm{H}_{2} \mathrm{O}
\end{aligned}
$$

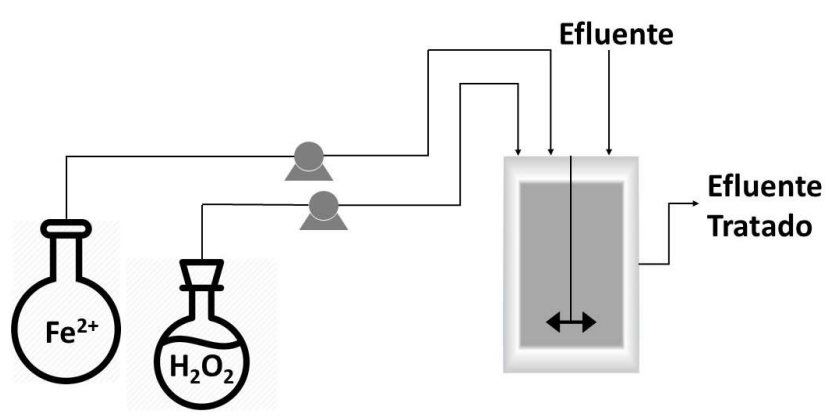

Figura 4: Processo Oxidativo Avançado - Processo Fenton

Silva $^{28}$ avaliou a descoloração e degradação de um corante (azul de metileno) através da reação Fenton 
heterogênea, utilizando como catalisadores os resíduos de aciaria, laminação e têmpera, em reator de leito fixo e em processo contínuo, e concluiu que o resíduo de têmpera apresentou cerca de $20 \%$ de descoloração do corente. O resíduo de laminação mostrou 77 \% e por último, o resíduo de aciaria apresentou $70 \%$. Após 60 min de reação, a ordem de atividade foi laminação $>$ aciaria $>$ têmpera.

\section{Conclusão}

Com o aumento de indústrias, em geral, nos dias atuais, a poluição fica evidente, e o descarte incorreto dos resíduos nos efluentes de águas, como em rios, lagos, represas, busca-se criar alternativas para descontaminar e descolorir estes meios ambientais. Uma das alternativas promissoras para isso são os POAs demonstrados nesse trabalho de revisão. Os Processos Oxidativos Avançados são um conjunto de métodos promissores para o tratamento de contaminantes persistentes e tóxicos de águas residuárias, considerando vários fatores, um deles é a eficiência na rápida mineralização de inúmeras espécies químicas de relevância ambiental.

\section{Agradecimentos}

Agradecimento ao suporte financeiro da CHAMADA PÚBLICA N ${ }^{\circ}$ 07/2016 - PROGRAMA DE APOIO A NÚCLEOS EMERGENTES - PRONEM/FAPEG/CNPq, CNPq/Universal 485518/2013-9; CAPES/PROCAD 2013/2998/2014, ao Instituto de Química da UFG/RC. Bolsas CNPq, CAPES e FAPEG.

\section{Referências}

1. Jovic, M.; Stankovic, M. D.; Manojlovic, D.; Andelkovic; Milic, A.. Study of the Electrochemical Oxidation of Reactive Textile Dyes Using Platinum Electrode. Int. J. Electrochem. Sci., 8168 - 183, 2013. [CrossRef]

2. Araújo, K. S.; Antonelli, R.; Gaydeczka, B.; Granato, A. C.; Malpass, G. R. P;. Advanced oxidation processes: a review of fundamentals and applications in the treatment of urban and industrial wastewaters. Revista Ambiente e Água; 11, 387-401, 2016. [CrossRef]

3. Ghaly, A., Ananthashankar, R., Alhattab, M., Ramakrishnan, V., Production, characterization and treatment of textile effluents: a critical review. J. Chem. Eng. Process Technol. 5 (1), 1-19, 2014. [CrossRef]

4. Rosa, J.M., Fileti, A.M., Tambourgi, E.B., Santana, J.C., Dyeing of cotton with reactive dyestuffs: the continuous reuse of textile wastewater effluent treated by Ultraviolet/Hydrogen peroxide homogeneous photocatalysis. J. Clean. Prod. 90, 60-65, 2015. [CrossRef]

5. Ayadi, I., Souissi, Y., Jlassi, I., Peixoto, F., Mnif, W., Chemical synonyms, molecular structure and toxicological risk assessment of synthetic textile dyes: a critical review. J Develop Drugs 5 (151), 2, 2016. [CrossRef]

6. Chequer, F.M.D., de Oliveira, G.A.R., Ferraz, E.R.A., Cardoso, J.C., Zanoni, M.V.B., de Oliveira, D.P., Textile Dyes: Dyeing Process and Environmental Impact, Eco-Friendly Textile Dyeing and Finishing. InTech, 2013.

7. FEAM - Fundação Estadual do Meio Ambiente. Guia Técnico Ambiental da Indústria Têxtil. minas gerais: 2014. Disponível em: < http://www.feam.br/images/stories/producao_sustentavel/ GUIAS_TECNICOS_AMBI ENTAIS/guia_textil.pdf $>$ Acesso em:17/10/2018.

8. Brillas, E.; Martínez-Huitle, C.A. Decontamination of wastewaters containing synthetic organic dyes by electrochemical methods. An updated review. Applied Catalysis B: Environmental; 166, 603-643, 2015. [CrossRef]

9. Martínez, C. A.; Rodrigo, M.A.; Sirés I.; Scialdone, O. Single and Coupled Electrochemical Processes and Reactors for the Abatement of Organic Water Pollutants: A Critical Review. Critical Review. Chemical Reviews; 24, 13362-13407, 2015. [CrossRef]

10. Gogate, P.R.; Pandit, A.B. A review of imperative technologies for wastewater treatment I: oxidation technologies at ambient conditions Advances In Environmental Research, Matunga, 8, 501-551, 2004. [CrossRef]

11. Brito, N. N. De.; Silva, V. B. M.. Processo Oxidativo Avançado e sua Aplicação Ambiental. Revista Eletrônica de Engenharia Civil; 1, 36, 2012. [CrossRef]

12. Domenech, X.; Jardim, W. F.; Litter, M. Procesos Avanzados de Oxidación para la eliminación de contaminantes, En: Eliminación de Contaminantes por Fotocatálisis Heterogénea. Editor: Miguel Blesa, Red CYTED VIII-G, 3, 26, 2001.

13. Giraldi T. R.; Swerts, J. P. ; Vicente, M. A.; de Mendonça, V. R.; Paris, E. C.; Ribeiro, C. Utilização de partículas de $\mathrm{ZnO}: \mathrm{Mn}$ para a degradação do azul de metileno por processo de fotocatálise. Cerâmica; 62, 345, 2016. [CrossRef]

14. Fioreze, M; Santos, E,P; Schmachtenberg, N..; Advanced Oxidative Processes: Fundamentals and Environmental Application. Revista Eletronica em Gestão, Educação e Tecnologia Ambiental, 18, 79, 2014. [CrossRef] 
15. Almeida, E.; Assalin, M.R.; Rosa, M.A.; Durán, N. Tratamento de efluentes industriais por processos oxidativos na presença de ozônio. Quím. Nova. 27, 5, 2004. [CrossRef]

16. Von Gurten, U.; Ozonization of drinking water: Part I. Oxidation Kinectics and product formation. Water Res. 37, 1443, 2003. [CrossRef]

17. Galvão, I. R.C.; Fischer, Lisete M. L.. VII Workshop Multidisciplinar Sobre Ensino e Aprendizagem Wea', Campo Limpo Paulista; 71, 2011.

18. Hernandez, R.; Zappi, M.; Colluci, F.; Jones, R. Comparing the performance of various advanced oxidation processes for treatment of acetone contaminated water. Journal Hazardous Materials, 92, 33, 2002. [CrossRef]

19. Marmitt, S.; Pirotta, L.V.; Stulp, S. Aplicação de fotólise direta e UV/H2O2 a efluente sintético contendo diferentes corantes alimentícios. Quimica Nova; 33,384-388, 2010. [CrossRef]

20. Oliveira, A.S, Saggioro; E.M.; Pavesi,T.; Moreira, J.C.; Vieira Ferreira, L.F. Solar photochemistry for environmental remediationAdvanced Oxidation Processes for Industrial Wastewar Treatment; 195-222, 2012. [CrossRef]

21. Henderson, M., A surface science perspective on $\mathrm{TiO} 2$ photocatalysis. Surface Science Reports, Richland; 66, 195-287, 2011. [CrossRef]

22. Rodrigues, M .H.M; Santos, L.M.; Gonçalves, R.F.; Santos, M.R.C.; Gurgel M.F.C., Godinho, M.J. Estudo da cinética de degradação do corante azul de metileno utilizando catalisadores de céria dopada com índio. The Journal of Engineering and Exact Sciences; 4, 2018. [CrossRef]

23. Gonçalves, M.C.P.; Padilha, S.R.R; Lopes, M.F.; Pari, E.C.; Giraldi, T.R. Aplicação de Nb2O5 comercial para descoloração do corante azul de metileno pelo processo de fotocatálise. Revista de Engenharia e Tecnologia. 9, 95-105, 2017. [CrossRef]

24. Youssef, Z.; Colombeau, L.; Yesmurzayeva, N.; Baros, F.; Acherar, S. Dye-sensitized nanoparticles for heterogeneous photocatalysis: Cases studies with $\mathrm{TiO} 2, \mathrm{ZnO}$, fullerene and graphene for water purification, Dyes and Pigments, Volume 159, 49-71, 2018. [CrossRef]

25. Rodrigues, M.H.M.; Sousa, P.A.R.; Borges, K.C.M.; Coelho, L.M.; Godinho, M. J. Enhanced degradation of the antibiotic sulfamethoxazoleby heterogeneous photocatal ysis using $\mathrm{Ce} 0,8 \mathrm{Gd} 0,2 \mathrm{O} 2-\delta / \mathrm{TiO} 2$ particles Journal of Alloys and Compounds, Volume 808, 5,151711, 2019. [CrossRef]

26. Borges, K. C. M.; Goncalves, R. F.; Rodrigues, M. H. M.; Godinho, M.J. Interface Influence on Photocatalytic Properties of Ag2MoO4/ZnO Heterojunctions. Orbital: The Eletronic Journal of Chemistry.11,124 - 127, 2019. [CrossRef]
27. Garcia, L.; Ferreira, T.; Gurgel, G.; Nascimento, R. M.; Rodrigues, M. H. M.; Godinho, M.J.; Bomio, M. R. D.; Motta, F. V. Effects of $\mathrm{MnO} 2 / \mathrm{In} 2 \mathrm{O} 3$ thin films on photocatalytic degradation 17 alphaethynylestradiol and methylene blue in water. Journal of Materials Science-Materials in Eletronics, 29, 12278 - 12287, 2018.[CrossRef]

28. Silva, A. G. M.; Rodrigues, T. S.; Dias, A.; Fajardo, H. V.; Gonçalves, R. F.; Godinho, M.; Robles-Dutenhefner, P. A. Ce1$\mathrm{xSm} \times \mathrm{O} 1.9-\delta$ nanoparticles obtained by microwave-assisted hydrothermal processing: an eficiente Application for catalytic oxidation of $\alpha$-bisabolol. Catalysis Science \& Technology. 4, p.814 821, 2014. [CrossRef]

29. Troque, B. F.; Eliziario, S. A.; Godinho, M.J.; Kiminami, R.H.A. Síntese in situ pelo método hidrotérmico de heteroestruturas de titanato-ferritas e sua atividade fotocatalítica. Cerâmica. 64, 248 253, 2018. [CrossRef]

30. Garrel, L.; Bonetti, M.; Tonucci, L.; Alessandro, N.; Bressan, M. Photosensitized degradation of cyclohexanol by Fe(III) species in alkaline aqueous media. Journal of Photochemistry and Photobiology A: Chemistry. 179, 193-199, 2006. [CrossRef]

31. Malato, S.; Fernández-Ibánez, P.; Maldonado, M.I.; Blanco, J.; Gernjak, W. Decontamination and disinfection of water by solar photocatalysis: Recent overview and trends. Catalysis Today. 147, 1-59, 2009. [CrossRef]

\section{Letícia G. Oliveira ${ }^{\text {* }}$, Fabrício H. Fernandes ${ }^{\prime}$, Weber D. Mesquita ${ }^{2}$, Mario G. Junior ${ }^{1,2}$, Maria R. de C. Santos' \& Maria F. do C. Gurgel ${ }^{1,2}$}

1 UFG-Universidade Federal de Goiás, Unidade Acadêmica Especial de Química- RC, Programa de Pós-Graduação em Química, CEP 75.704- 020, Catalão, GO, Brasil

2 UFG- Universidade Federal de Goiás, Unidade Acadêmica Especial de Física- RC, Programa de Pós-Graduação em Ciências Exatas e Tecnológica (PPGCET), CEP: 75.704- 020, Catalão, GO, Brasil

* E-mail: leticinhaguioliveira@hotmail.com 
Artigo Geral 11 